\title{
Diacronie
}

Studi di Storia Contemporanea

$\mathrm{N}^{\circ} 20,4 \mid 2014$

II diritto militante

\section{Epistemologie del Sud: il postcolonialismo e lo studio delle relazioni internazionali}

Antônio Manoel Elíbio Júnior e Carolina Soccio Di Manno De Almeida

Traduttore: Jacopo Bassi

(2) OpenEdition

Journals

\section{Edizione digitale}

URL: http://journals.openedition.org/diacronie/1771

DOI: 10.4000/diacronie.1771

ISSN: 2038-0925

\section{Editore}

Association culturelle Diacronie

Notizia bibliografica digitale

Antônio Manoel Elíbio Júnior e Carolina Soccio Di Manno De Almeida, « Epistemologie del Sud: il

postcolonialismo e lo studio delle relazioni internazionali », Diacronie [Online], № 20, 4 | 2014 documento 13, online dal 01 décembre 2014, consultato il 10 décembre 2020. URL : http:// journals.openedition.org/diacronie/1771 ; DOI : https://doi.org/10.4000/diacronie.1771 


\title{
Diacronie
}

\section{3/}

\section{Epistemologie del Sud: il postcolonialismo e lo studio delle relazioni internazionali}

\author{
Antônio Manoel ELÍBIO JÚNIOR, Carolina SOCCIO DI MANNO DE ALMEIDA*
} traduzione di Jacopo BASSI

L'articolo affronta lo sviluppo degli studi postcoloniali, contestualizzando i dibattiti teorici avvenuti nel campo delle relazioni internazionali. A partire dalla seconda metà del XX secolo, in concomitanza con i processi di decolonizzazione e globalizzazione, iniziarono a prendere colpo una serie di analisi e studi influenzati da questo nuovo scenario mondiale e tracciarono una nuova corrente teorica: il postcolonialismo.

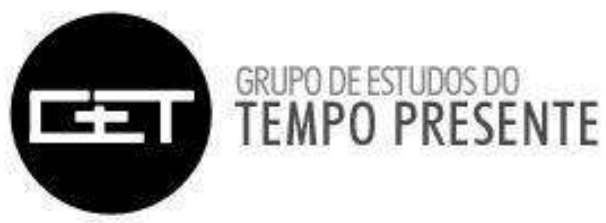

Il presente articolo viene pubblicato in traduzione italiana per gentile concessione di Cadernos do Tempo Presente nell'ambito del progetto di collaborazione tra le due riviste.

\section{L'interazione fra relazioni internazionali e studi postcoloniali}

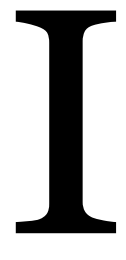

1 Ventesimo secolo è stato lo scenario di profonde e multiformi trasformazioni nella totalità del globo, di carattere politico, culturale, economico e sociale, verificatisi con una rapidità senza precedenti nella storia del mondo. Questo processo portò a una nuova divisione internazionale del lavoro, la transnazionalizzazione della produzione e del consumo, la frammentazione e la diffusione delle culture, accompagnato dal multiculturalismo, la dissoluzione delle frontiere e la pretesa multipolarità di un mondo fino ad allora bipolare e, prima di quel momento, contrassegnato dalla presenza degli imperi. In questo contesto è necessario 
riconoscere l'importanza della decolonizzazione dell'Africa e dell'Asia come momento rilevante dal punto di vista geopolitico, dal momento che segnala un drastico mutamento nello scenario internazionale. La liberazione di più della metà della popolazione mondiale dal dominio diretto dei paesi europei e la diaspora dei popoli di questi luoghi sviluppatasi lungo flussi migratori che riproducevano le rotte coloniali richiede una riflessione crescente sui regimi coloniali e le conseguenze ancora sconosciute dei fenomeni che emergevano nel periodo definito "postcoloniale". A partire da ciò, una serie di analisi e studi rivolti a questo nuovo scenario mondiale, sorto dai residui del colonialismo, cominciarono a prendere corpo e a tratteggiare una nuova corrente teorica, il postcolonialismo.

Allacciando diverse aree delle scienze umane e sociali, il postcolonialismo è stato per lungo tempo ignorato come disciplina nell'ambito delle Relazioni Internazionali, rimanendo assente dai grandi dibattiti teorici e dai corsi di studio, così come dai manuali e dalle riviste specializzate. Questa sconcertante mancanza di dialogo tra gli studi postcoloniali e le relazioni internazionali può essere dovuta a due ragioni fondamentali: da un lato, al fatto che gli studi postcoloniali erano legati ai cosiddetti studi culturali ed incontrarono una certa resistenza a superarlo; tuttavia muoversi tra gli studi di economia internazionale è prodromico all'elaborazione di una critica al capitalismo globale. Dall'altro, al rifiuto da parte dell'establishment dei teorici delle relazioni internazionali, di orientamento eminentemente protezionista e conservatore, di un approccio che, scientificamente e politicamente, sovverte la maggior parte - per non dire la totalità - dei suoi presupposti principali.

Tuttavia, in questo primo decennio del XXI secolo, la diffusione del postcolonialismo nelle scienze sociali, assieme allo sforzo dei teorici provenienti dalle più disparate aree della conoscenza ha portato ad un approfondimento del tema, articolato in differenti campi, ed è possibile percepire questo sforzo nelle relazioni internazionali. Si sta costituendo un nucleo di accademici che ha sviluppato linee di ricerca e prodotto pubblicazioni che collocano gli studi postcoloniali e le questioni che solleva, nel solco di questa disciplina.

Il dibattito rimane comunque in una posizione marginale nell'ambito della disciplina, la questione che poniamo inizialmente è dunque la rilevanza dell'approccio postcoloniale per le relazioni internazionali esplorando le potenzialità e le difficoltà di questo dialogo. Per questa ragione abbiamo bisogno di mettere a fuoco le principali questioni sollevate dal postcolonialismo per giungere alla conclusione che questo dialogo richiede una riconfigurazione radicale del complesso teorico e della pratica politica delle relazioni internazionali, sostenuta da tre orientamenti principali: la 
revisione della storia delle relazioni internazionali, il rovesciamento di concetti centrali come potere e Stato-nazione e, infine, il tornante epistemologico nella "geopolitica della conoscenza”.

Lo studio delle relazioni internazionali non è assolutamente recente: al contrario, considerazioni ed elaborati sul contesto internazionale sono ricorrenti nelle scienze sociali, principalmente nella storia e nella scienza politica. La guerra del Peloponneso di Tucidide è considerata da molti teorici come un'opera paradigmatica in questo campo di studi, al pari di quelle di Niccolò Machiavelli e Thomas Hobbes per la teorizzazione moderna del sistema internazionale e di interazione tra gli Stati ${ }^{1}$. Tuttavia è stato solamente durante gli anni Cinquanta, Sessanta e Sessanta del XX secolo che le relazioni internazionali si sono ritagliati una certa autonomia, separandosi dalla scienza politica e costituendo un proprio filone narrativo ${ }^{2}$.

Lo studio delle relazioni internazionali rimane strettamente legato alla realtà politica internazionale: le sue origini risalgono agli anni immediatamente successivi alla Prima guerra mondiale, in cui nacque con il chiaro intento di evitare il verificarsi delle circostanze che avrebbero potuto portare ad un'altra guerra. Come ricorda Halliday3 fu in questo periodo che vennero creati in Gran Bretagna, nelle università, le prime cattedre e i dipartimenti relativi a questa materia: nella Aberystwyth, nella London School of Economics a Oxford e, nel mondo non accademico nel Royal Institute of International Affairs, votato a proporre e portare avanti politiche pubbliche. Questa tendenza fu perseguita dagli Stati Uniti che, contemporaneamente, crearono cattedre nelle università statunitensi e il Council of Foreign Relations. Come afferma Hoffman 4 , modernamente lo studio delle relazioni internazionali negli Stati Uniti si è profondamente legato al problema, tanto politico quanto accademico, di come il Paese avrebbe dovuto amministrare la sua egemonia internazionale contrastando la costante minaccia sovietica, in modo che gli studiosi delle relazioni internazionali ponessero le basi teorico-scientifiche per una "nuova diplomazia nordamericana", di tendenze imperialiste, che potesse mettere gli studi strategico militari in una posizione centrale ${ }^{5}$. Da allora la concettualizzazione della forza, del potere e delle sue dinamiche, interessi tipicamente nordamericani, si trasformarono in fondamenti della teoria delle relazioni

${ }^{1}$ INAYATULLAH, Naeem, BLANEY, David L., International Relations and the Problem of the Difference, London Routledge, 2004, p. 45.

2 HOFFMAN, Stanley, An American Social Science: International Relations, in DER DERIAN, J. (org.), International Theory: Critical Investigations, London, MacMillan, 2005, p. 134.

3 HALLIDAY, Fred, Rethinking international relations, London, MacMillan, 1994.

4 HOFFMAN, Stanley, An American Social Science: International Relations, in DER DERIAN, James (org.), International Theory: Critical Investigations, London, MacMillan, 2005.

5 HOFFMAN, Stanley, An American Social Science: International Relations, in DER DERIAN, James (org.), International Theory: Critical Investigations, London, MacMillan, 2005, p. 135. 
internazionali successivamente divenuta ad una monocultura realista, che per questa ragione - e per un lungo lasso di tempo - ha detenuto il monopolio della discussione in questo campo di studi.

L'ondata liberale che investì la teoria delle relazioni internazionali nei primi decenni del XX secolo incentrata sul diritto internazionale e sulla sua connotazione al mutare del sistema internazionale per via istituzionale-legale, ricevette l'etichetta ampiamente diffusa e accettata di "idealista"; venendo considerata ingenua, di poca rilevanza scientifica è stata accusata di essere alla base del ritardo con cui le relazioni internazionali sono emerse come scienza. Ad Edward Carr toccò, con la sua opera fondamentale Twenty years' Crisis $^{6}$, l'incombenza di inaugurare la trattazione scientifica, obiettiva ed empirica di un contesto politico definito come una lotta per il potere oramai incompatibile con le sue proposizioni normative. Tuttavia fu solamente negli Stati Uniti del dopoguerra, che erano allora emersi come superpotenza, che le relazioni internazionali si stabilirono sulla base del realismo politico di Hans Morgenthau, un concetto sviluppato nel suo Politics among Nations7. Proprio quest'ultimo nella sua opera cercò di identificare quelle regolarità che avrebbero potuto fornire una prevedibilità nel comportamento degli Stati basandosi principalmente sui concetti di potere e di interesse nazionale. Fu proprio l'ambizione di Morgenthau di fissare i principi irrefutabili della politica internazionale, unita alla sua argomentazione molto fragile a generare un dibattito e una serie di letture volte a reagire proprio nei confronti dell'approccio realista. In questo modo il campo di studi si consolidò in rapporto o in contrapposizione con il realismo e, in una certa misura, continua a farlo.

Il cosiddetto secondo dibattito delle relazioni internazionali si svolse tra gli approcci definiti "tradizionalisti” che ricavavano alcuni contributi dal behaviorismo, almeno per quel che afferma riguardo alle metodologie che dovrebbero essere impiegate in questo campo, ma non apportarono contributi all'estensione della materia di competenza della disciplina, che continuò in larga scala a far riferimento ai dettami iniziali del realismo. Secondo Hedley Bull fu solo al termine degli anni Settanta, con il movimento pacifista mondiale, la fine di Bretton Woods, la percezione di una crescente interdipendenza tra le economie nazionali e certi movimenti politici di contestazione del Terzo Mondo, che la smisurata attenzione nei confronti delle dinamiche di potere e forza diede vita ad altre problematiche, legate soprattutto all'accresciuta importanza della politica

6 CARR, Edward H.,Twenty years' crisis 1919-1939: an introduction to the study of international relations, London-New York, MacMillan-St Martin's Press, 1946.

7 MORGENTHAU, Hans J., Politics among nations : the struggle for power and peace, New York, Alfred A. Knoff, 1952. 
economica internazionale ${ }^{8}$. Questa apertura nel campo teorico, intensificatasi negli anni Ottanta, fece sì che fossero riconosciute tematiche che fino a quel momento erano state messe al margine dalle stringenti formulazioni del Realismo, come gli studi sulla pace le analisi di politica estera e l'importanza assunta dall'economia politica internazionale.

In questo senso Halliday afferma che dopo una fase "protezionista", recentemente le relazioni internazionali si stanno aprendo a contributi provenienti da altri campi del sapere che non erano tradizionalmente legati alla sua sfera teorica9. Il risultato ottenuto è che dalla fine degli anni Ottanta e, soprattutto, con le questioni sollevate dal "terzo dibattito" delle relazioni internazionali sono stati introdotti in questo campo i Critical Legal Studies (Crits), il costruttivismo, il postmodernismo e il femminismo, e abbiamo assistito all'emergere di quegli itneressi, di quelle critiche e di quelle operazioni di decostruzione che fino ad allora erano state escluse dal campo teorico della disciplina. Tuttavia è necessario mettere in evidenza come la maggior parte della produzione teorica risponda ad una prospettiva realista e sia riconducibile ai suoi interessi tradizionali, ancorché riadattati ai tempi ${ }^{10}$. Al di fuori degli Stati Uniti si è fatta sentire la dipendenza teorico-strutturale, consolidatasi nel corso dei decenni, che ha impedito una teorizzazione indipendente o, se vogliamo, non egemonica, delle relazioni internazionali, allora legata a livello globale, al linguaggio del potere, dello StatoNazione e dell'interesse nazionale. Slater osserva come benché i teorici del Nord possano concedersi il lusso di disconoscere o ignorare le teorie del Sud del mondo, mentre lo stesso non avvenga con la controparte del Sud, la cui teorizzazione è sempre stata in accordo, rifiuto o opposizione alla teoria sociale del Nord ${ }^{11}$.

Halliday sottolinea come, forse, tra tutte le scienze sociali le relazioni internazionali, siano quelle che più si sono tenute lontane dal marxismo e dalle questioni di genere; ciò ha comportato una serie di problemi che hanno contribuito al loro indebolimento come l'incapacità di mettere in dubbio criticamente il complesso delle relazioni Nord-Sud e le stesse strutture globali di sfruttamento ${ }^{12}$. Non per niente, le relazioni internazionali appaiono anche adesso come l'area delle scienze sociali che si mostra più indifferente nei confronti degli approcci postcoloniali. Questa distanza si rende ogni volta più

\footnotetext{
8 BULL, Hedley, The theory of International Politics, 1919-1969, in DER DERIAN, James (ed. by), International Theory: Critical Investigations, London, MacMillan, 2005, p. 36.

9 HALLIDAY, Fred, Rethinking international relations, London, MacMillan, 1994, p. 26.

1o DER DERIAN, James, Introduction: Critical Investigation, in DER DERIAN, James (org.), International Theory: Critical Investigations, London, MacMillan, 2005, p. 56.

11 SLATER, David, «Post-colonial questions for global times», in Review of international Political Economy, 5, 4/1998, p. 28.

${ }^{12}$ HALLIDAY, Fred, Rethinking international relations, London, MacMillan, 1994, p. 130.
} 
deleteria in un mondo che è maggioritariamente non-occidentale, in cui le asimmetrie di potere e ricchezza sono aumentate e in cui i nuovi attori, non-statali o subnazionali, iniziano a farsi sentire e a imporre nuove necessità. Debitrice nei confronti del predominio teorico anglo-americano, la disciplina si è costruita attorno ad una molteplicità di concetti, presupposti e interessi tipicamente occidentali e che non avevano un grande significato in altri contesti, Paesi e regioni che ancora non erano considerabili come attive nell'ambito politico e analitico delle relazioni internazionali ${ }^{13}$. $\grave{E}$ in questo contesto che gli studi postcoloniali si trasformano in uno strumento essenziale, teorico e politico, per identificare e inquadrare da un punto di vista teorico la grande quantità di attori, dinamiche ed esigenze che ruotano intorno allo Stato, al di fuori delle concezioni egemoniche di oggi e di un tempo.

\section{Epistemologie del Sud}

Quali sarebbero, perciò, i principali fondamenti teorici dell'approccio postcoloniale? In primo luogo è importante mettere in luce che il "post" del postcoloniale non significa, in termini assoluti, una rottura con il periodo precedente, quello coloniale, e neppure un suo superamento, dal momento che «la fine del colonialismo in quanto relazione politica non comportò la fine del colonialismo inteso come relazione sociale, mentalità e forma di sociabilità autoritaria e discriminatoria» ${ }^{14}$. L'accezione fondativa degli studi postcoloniali presuppone una revisione critica del passato considerato nei termini della modernità occidentale e la sua identificazione con un presente ancora permeato da una serie di narrative, pratiche, rappresentazioni e relazioni politiche che confluiscono nella perpetuazione della distribuzione asimmetrica del potere e della ricchezza a livello globale.

Come sostiene Boaventura de Sousa Santos, uno dei fondamenti teorici principali dell'approccio postcoloniale è «la diluizione della frontiera tra critica e politica»15. In maniera più esplicita Said afferma:

Ciò che qui tengo a sottolineare è che il consenso generalizzato ,nelle società liberali, sulla nozione che il "vero" sapere sia fondamentalmente non politico (e, inversamente, che un sapere politico non sia "vero" sapere) oscura l'enorme importanza, anche se spesso difficile a descriversi e

\footnotetext{
13 HOFFMAN, Stanley, op. cit., p. 136.

14 SANTOS, Boaventura de Sousa, Entre o próspero e o Caliban: Colonialismo, PósColonialismo e interidentidade, in RAMALHO, Irene, RIBEIRO, António Sousa (orgs.), Entre ser e estar:Raízes, Percursos e Discursos da Identidade, Porto, Afrontamento, 2001, p. 38.

15 Ibidem, p. 39.
} 
dimostrarsi, dalle circostanze politiche per il prodursi del sapere umano in ogni sua forma ${ }^{16}$.

Riconoscendo le intricate relazioni sapere-potere soggiacenti ad ogni pratica accademica, gli studi postcoloniali propone una critica - un sapere - che sia cosciente della sua funzione politica e che sappia opporsi all'asimmetrica distribuzione globale del potere e a tutte quelle ingiustizie che essa porta con sé. In questo senso gli studi postcoloniali devono conseguire lo scopo di ottenere l'implosione dei discorsi egemonici coloniali/occidentali che, soprattutto attraverso la retorica della modernità, considerano naturali le disuguaglianze fra paesi, classi, razze e popoli ${ }^{17}$.Quando Gayatri Spivak pone la domanda «Can the subaltern speak?», lega la messa ai margini dei gruppi e della retorica subalterna al fatto che siano stati ridotti al silenzio: individua nella parola la conditio sine qua non per il sovvertimento della subalternità ${ }^{18}$. Per questa teorica indiana lavorare perché emerga questa parola richiede un'attività politica che oltrepassa la retorica accademica, segnando un vero e proprio impegno della critica postcoloniale in favore dei "subalterni della Terra". Boaventura de Sousa Santos si appropria di questa volontà di privilegiare la subalternità in quanto luogo di enunciazione da cui gli studi postcoloniali possano emergere, ponendo questa prospettiva nella premessa secondo la quale i margini e le periferie sono i loci (o i luoghi) di enunciazione privilegiati per identificare e sovvertire le strutture di potere del sapere ${ }^{19}$.

Un altro punto caro agli studi postcoloniali è il rifiuto dell'essenzialismo. La critica dei regimi coloniali ci mostra come il potere e la retorica coloniale si siano costituiti facendo ricorso ad una "violenza epistemica", che forgiò il soggetto colonizzato in quanto Altro rispetto al colonizzatore. Del resto il colonizzatore e - in un'accezione più ampia - l'Occidente hanno costruito loro stessi in relazione con il Self, a partire dal quale il resto del mondo è subalterno/reso subalterno. Il rifiuto degli studi postocoloniali di queste opposizioni binarie, a partire dalle quali le narrative coloniali hanno realizzato tutte le forme di razzismo, si verifica nella misura in cui l'intento è

${ }^{16}$ SAID, Edward W., Orientalismo. L'immagine europea dell'Oriente, Milano, Feltrinelli, 2006, p. 19.

${ }_{17}$ BHABHA, Homi K., The location of culture, London, Routledge, 1994, p. 17.

${ }^{18}$ SPIVAK, Gayatri Chakravorty, Can the subaltern speak?, in ASHCROFT, Bill, GRIFFITHS, Gareth, TIFFIN, Helen, The postcolonial studies reader, London, Routledge, 1988, p. 54.

19 SANTOS, Boaventura De Sousa, Do Pós-Moderno ao Pós Colonial. E para além de um e outro, Coimbra, Centro de Estudos Sociais, Universidade de Coimbra, 2004, p. 12, URL:

< http://www.ces.uc.pt/misc/Do_pos-moderno_ao_pos-colonial.pdf > [consultato il 16 novembre 2014]. 
quello dicercare identità complesse e multisfaccettate, ma coerenti con la realtà politica.

\section{Considerazioni finali}

Gli studi postcoloniali contengono al loro interno la critica dei presupposti epistemologici su cui si afferma la retorica della modernità e, conseguentemente, della superiorità europea. Spivak considera la subalternità come un prodotto delle penetranti relazioni di "violenza epistemica" causate dall'imposizione coloniale di un ordine scientifico e di un sistema legale ${ }^{20}$. In questo modo la celebrazione della modernità e dei suoi presupposti si iscrive in una relazione di potere regolata dalla differenza coloniale fra quelli che "pensano, quindi, esistono" e quelli che non esistono e non sono in ragione del fatto che sono oggetto di un'egemonia.

Il consolidamento della critica postcoloniale in un corpus teorico potrebbe trasformarlo facilmente in generalizzazioni: si incorrerebbe negli stessi essenzialismi e generalizzazioni che questa corrente teorica ambisce a combattere. Benché possa essere concepito come un "sistema" di caratteristiche fondamentali condivise, il colonialismo non si impose in maniera omogenea nei differenti contesti storici, sociali e geografici su cui estese il suo dominio. Per questo motivo non è possibile fare generalizzazioni, così come questo non rappresenterebbe un arricchimento per il dibattito all'interno della corrente teorica.

Il post-colonialismo può fornire un contributo nell'ambito delle analisi culturali, sociali e politiche di qualche parte del mondo, principalmente per ciò che afferma riguardo alle asimmetrie del potere. Non volendo perdere di vista le potenzialità analitiche e politiche dell'approccio postcoloniale e allo stesso tempo cercando di inquadrare il problema della sua applicabilità, Boaventura de Sousa Santos propone un "postcolonialismo circoscritto", che prenda in conto la grande specificità di ogni contesto storico dove i differenti colonialismi sono utili alla comprensione dei diversi contesti postcoloniali ${ }^{21}$.

${ }^{20}$ SPIVAK, Gayatri Chakravorty, op. cit., p. 57.

${ }^{21}$ SANTOS, Boaventura De Sousa, op. cit, p. 34. 


\section{* Gli autori}

Antônio Manoel Elíbio Júnior ha conseguito un dottorato in Storia sociale presso l'Universidade Estadual de Campinas-UNICAMP e un post-dottorato in Scienze politiche presso l'Universidade Federal de Pernambuco-UFPE. Attualmente è professore nel Dipartimento di Storia nell'Universidade Estadual da Paraíba-UEPB.

URL: < http://www.studistorici.com/progett/autori/\#Elibio-Junior >

Carolina Soccio Di Manno de Almeida è laureata in Scienze politiche nell'Universidade Federal de Pernambuco.

URL: < http://www.studistorici.com/progett/autori/\#Soccio-DiManno-deAlmeida >

\section{Per citare questo articolo:}

ELÍBIO JÚNIOR, Antônio Manoel, SOCCIO DI MANNO DE ALMEIDA, Carolina, «Epistemologie del Sud: il postcolonialismo e lo studio delle relazioni internazionali», Diacronie. Studi di Storia Contemporanea : II diritto miltante, 29/12/2014,

URL:<http://www.studistorici.com/2014/12/29/elibio-soccio_numero_20/ >

Diacronie Studi di Storia Contemporanea $\widehat{\beta}$ www.diacronie.it

Risorsa digitale indipendente a carattere storiografico. Uscita trimestrale. redazione.diacronie@hotmail.it

Comitato di redazione: Jacopo Bassi - Luca Bufarale - Elisa Grandi - Deborah Paci - Fausto Pietrancosta - Matteo Tomasoni - Luca Zuccolo 